University of Nebraska - Lincoln

DigitalCommons@University of Nebraska - Lincoln

\title{
Research Needs Concerning Non-target Impacts of Biological Control Introductions
}

\author{
K.R. Hopper \\ USDA-ARS
}

Follow this and additional works at: https://digitalcommons.unl.edu/usdaarsfacpub

Part of the Agricultural Science Commons

Hopper, K.R., "Research Needs Concerning Non-target Impacts of Biological Control Introductions" (2001). Publications from USDA-ARS / UNL Faculty. 478.

https://digitalcommons.unl.edu/usdaarsfacpub/478

This Article is brought to you for free and open access by the U.S. Department of Agriculture: Agricultural Research Service, Lincoln, Nebraska at DigitalCommons@University of Nebraska - Lincoln. It has been accepted for inclusion in Publications from USDA-ARS / UNL Faculty by an authorized administrator of DigitalCommons@University of Nebraska - Lincoln. 


\title{
Research Needs Concerning Non-target Impacts of Biological Control Introductions
}

\author{
K.R. Hopper
}

\author{
Beneficial Insect Introductions Research Unit, Agricultural \\ Research Service, United States Department of Agriculture, \\ 501 South Chapel Street, Newark, DE 19713, USA
}

\begin{abstract}
Two workshops were held in 1999 on research needs concerning non-target impacts of biological control introductions. One took place on the Internet for 9 months, and the other in Montpellier, France, for 1 day. Altogether, over 200 researchers from over 30 countries participated, representing a wide variety of viewpoints on the issues involved. The deliberations of these workshops are summarized here. Both workshops highlighted the need for retrospective studies to: (i) identify cases of significant non-target impacts; (ii) explore mechanisms involved when such impacts are found; (iii) evaluate the reliability of host-range testing protocols; and (iv) determine whether and under what circumstances host ranges of biological control agents have evolved subsequent to introduction. Both workshops also emphasized the need for better understanding of the mechanisms underlying host specificity. Finally, both workshops recognized the desirability of collaboration among ecologists, economists, sociologists and biological control practitioners in research to assess and reduce the potential for non-target impacts of biological control introductions.
\end{abstract}

\section{Introduction}

This chapter summarizes the deliberations of two workshops: one sponsored by the Agricultural Research Service, United States Department of Agriculture, and held on the Internet during January-September 1999; 
the other sponsored by the International Organization for Biological Control (IOBC) and held in Montpellier, France, on 20 October 1999, to conclude a 3-day conference on the subject. The Internet workshop was on research needs for assessing and reducing non-target impacts of biological control introductions. The Montpellier workshop was on research needs for evaluating non-target ecological effects of biological control, and thus was intended to cover not only introductions of exotic species but also biological control by augmentation and conservation of natural enemies. However, the emphasis was on non-target impacts of introductions for biological control.

\section{Internet workshop on 'Research needs for assessing and reducing non-target impacts of biological control introductions'}

Over 200 people from 28 countries subscribed to the mail-list through which the workshop was conducted; 49 participants posted 150 messages. An archive of these messages (with contributor names) is available at <http://www.udel.edu/entomology/khopper $>$. The emphasis was on arthropods introduced to control weeds and arthropods. Although there were a few postings on tangential issues (e.g. the biology of particular natural enemies, transgenic plants, the definition of biological control), most of the postings can be grouped under four germane topics: (i) what sorts of non-target impacts should concern us; (ii) host range evaluation; (iii) predicting impacts of biological control introductions versus other management options; and (iv) retrospective studies to assess actual impacts. The ideas summarized below are the contributions of 49 participants.

\section{What sorts of non-target impacts should concern us?}

Agreement is not complete on the species that should be considered when evaluating non-target impacts. Some have argued that impacts on all species should be considered equally; others hold that some species should receive special consideration (e.g. endangered species, species that provide crucial ecosystem services, species that provide economic benefits to society), and others hold that only such species should be of concern and impacts on others should be ignored. Although most agree that we should be concerned about population-level impacts rather than attacks on individuals, the magnitude and spatio-temporal scale of population-level impact that should concern us are not agreed upon. Depending on how impact is defined, its measurement may be extremely difficult. Field collection of hosts and exposure of sentinel hosts give impact on individuals which is hard to translate into impact on populations. Some argue that 
indirect impacts of arthropod introductions on parasitoids of non-target hosts may be larger than impacts on the hosts themselves. This is because parasitoids may be more liable to extinction than their hosts (LaSalle and Gauld, 1992; Unruh and Messing, 1993). However, such indirect impacts may be more difficult to measure than direct impacts on non-target hosts or prey. Not all impacts on non-target species need be considered detrimental. For example, introduced biological control agents might reduce the abundance of common native species that compete with or prey upon endangered native species, so that the introduction would decrease the extinction probability of the endangered species. Ultimately, the definition of non-target impact and how such impact is valued is a societal decision, but researchers attempting to evaluate non-target impacts need working definitions for design of surveys and experiments. Such definitions may be system specific, but it would be useful to agree upon guidelines for the sorts of impacts to consider.

\section{Host range evaluation}

Host range evaluation is usually the first, and often the only, step in predicting risks of non-target impacts. Some argue that the literature provides a good first screening for host range, especially for distinguishing candidates with broad versus narrow host ranges. Others argue that misidentifications in the literature, and especially the older literature, often cause problems of spurious host records. Some of these problems may be avoided by weighing the quality of literature data on host range, e.g. by taking into account numbers of individuals examined, percentage attack and spatio-temporal extent of studies. Laboratory or field experiments can be used to confirm data from field collections. However, physiological/behavioural host range measured in the laboratory and ecological host range measured in the field often differ. Host range evaluation in the area of origin can help with this problem by allowing comparison of field collections and laboratory measurements. An inherent assumption is that host range in the area of origin is a reasonably good predictor of host range in the area of introduction. Good design of experiments on host range usually involves system-specific factors. For example, the value of choice versus no-choice testing depends on the metapopulation dynamics of the system. If pests and non-target species commingle, or if a biological control agent can disperse from patches of pests to patches of non-targets, choice testing may be crucial. On the other hand, if biological control agents are likely to find themselves in areas without the target pest, no-choice testing may be more relevant. Some argue that host-range evolution poses significant problems for prediction of the host range of introduced natural enemies. Others argue that there is little evidence for host-range evolution after introduction, especially for herbivores introduced to control weeds (Marohasy, 1996). High levels of 
feeding and development on non-target species by a few individuals of a species may represent a greater risk of subsequent adaptation to the non-target than low levels of feeding or development by most individuals. This means that host-range testing should track the feeding and development of individuals and families. This will at least allow estimation of the likelihood of shifts in frequencies of genes/genotypes after introduction, although it will not provide information on the likelihood of novel genes arising. Ultimately, a deeper understanding of mechanisms affecting host specificity would allow better prediction of host ranges. Problems with host range screening of entomophages (difficulty of obtaining and rearing non-target species, problems with anomalous responses in the laboratory) may make such understanding particularly important for introductions against insects.

\section{Predicting impacts of biological control introductions versus other management options}

Host range testing is the most tractable approach to evaluating risks of impacts on non-target species. If one can show that a candidate for introduction will not attack any non-target species in the area of introduction, risk of non-target impacts is low. However, even in this case, indirect impacts may occur, e.g. if the target pest supports native species in some way. Furthermore, proving no potential for feeding or development on non-target species is difficult because one cannot test all possible non-target species and because host range may evolve after introduction. What is perhaps more important is that although many candidates for introduction have narrow host ranges, few are monospecific. The inclusion of species in the host range of an introduction candidate is often not simply dichotomous, with a list of species that are suitable for feeding and development neatly separated from those that are not suitable. Thus, several non-target species may be exposed to some reduced risk of attack compared with the target, and evaluation of the risks of significant impacts on these less suitable non-targets requires translation of attack on individuals to effects on populations. But there are major problems with predicting such impacts for target pests, let alone for non-target species. Furthermore, most agree that non-target impacts should be weighed not only against the value of controlling the target pest, but also against the risks versus benefits of other management options (Carruthers and Onsager, 1993; McEvoy, 1996; Simberloff and Stiling, 1996; Ricciardi et al., 1998). If introductions are not made, resources devoted to other strategies may lead to increased biological control by natives (conservation, switching of generalists) or other successful control of the target. On the other hand, the pest may continue unabated and cause enormous economic or environmental damage. Unfortunately, we do not yet have the theory and methods that would allow 
quantitative comparison of the risks versus benefits of all management options. Although many debate whether we can develop methods for predicting which natural enemies are likely to be most effective, most researchers agree that pre-release studies in the areas of origin and introduction are needed to determine the value of controlling the pest, whether biological control introductions are likely to prove useful, and whether non-target impacts are likely. Mathematical models of the population dynamics of introductions may provide a common framework for comparing various systems and to handle the complexity of the potential interactions in most systems. The problem with modelling real systems is that so little is known that describing system structure, let alone estimating model parameters, may prove impossible at present and for the foreseeable future.

\section{Retrospective studies to assess impacts}

All discussion participants recognized the need for retrospective studies of previous introductions and their impacts. Such studies can provide the testing ground for predictions about non-target impacts, whether these are based on estimates of host range from field collections or laboratory experiments or on mathematical models of system dynamics. However, it is crucial to assess impacts in the light of host range testing and predictions made prior to introduction so that methods for assessing impacts prior to introduction can be evaluated. Releases have occurred when some level of attack on non-target species was possible, but considered less risky than alternatives for management of the pest (Kok et al., 1992; Blossey et al., 1994; Hasan and Delfosse, 1995; Hill and Hulley, 1995; Olckers et al., 1995; Willis et al., 1995). Impacts on non-target species and evolution of host range have rarely been evaluated for these cases in the past, but these in troductions provide excellent opportunities for study. Some examples are:

1. Chrysolina quadrigemina feeding on Hypericum calycenum, an exotic plant extensively used as a ground cover in northern California, and on a native Hypericum species (Andres, 1985);

2. Rhinocyllus conicus introduced against Carduus nutans and feeding on non-target Cirsium spp. (Turner et al., 1987; Louda et al., 1997; see Gassmann and Louda, Chapter 8 , this volume);

3. Biological control agents for introduced weedy Solanum spp. and feeding on aubergine Solanum melongena in South Africa (Hill and Hulley, 1995; Olckers et al., 1995);

4. Galerucella spp. released against introduced Lythrum salicaria and feeding on native Lythrum alatum and Decodon verticillatus (Kok et al., 1992; Malecki et al., 1993; Blossey et al., 1994);

5. An eriophyid mite feeding on native Hypericum species in Australia (Willis et al., 1995); 
6. Tyria jacobaeae introduced against Senecio jacobaea feeding on native Senecio spp. (Diehl and McEvoy, 1990);

7. Diachasmimorpha tryoni imported to Hawaii for fruit fly control parasitizing non-target tephritid Eutreta xanthochaeta imported for biological control of lantana in Hawaii (Duan et al., 1997).

Several of these introductions are now being studied to assess non-target impacts, e.g. R. conicus (Turner et al., 1987; Louda et al., 1997; see Gassmann and Louda, Chapter 8, this volume), Gallerucella spp. (Malecki et al., 1993) and D. tryoni (Duan et al., 1997). But there are problems with the spatial and temporal scale of impacts versus our capacity to study introductions over large areas and long periods. All natural systems are dynamic, and introductions in particular may take a long time to reach equilibrium or at least a relatively steady state. Such dynamic systems present a moving target so that one may need to wait until an introduced agent is established over a broad area for a long time before evaluating the impacts of introduction. Ten years is often used as the time horizon for evaluating impacts of introductions on targets, but this is just a rule of thumb and may not even be applicable for target impacts, let alone non-target impacts. Such long time horizons and large spatial scales often put evaluations of target, as well as non-target, impacts beyond the resources available to most researchers. Unless thorough sampling was done before introduction, studying introduced species that have established over a large area for a long time means that potential control sites without the introduced species will be difficult or impossible to find. On the other hand, the slow rate of spread and patchy distribution of many introduced agents means that control sites without the introduced agent are often available long after introduction. A major problem with extensive surveys for non-target impacts is that negative evidence is hard to quantify and publish. Showing in a convincing way that small but significant impacts have not occurred is much more difficult and time-consuming than showing that large impacts have occurred. Although both conservationists and biological control practitioners regard with dismay illegal introductions and temporal and geographical differences in societal views about acceptable procedures for biological control introductions, differences in procedures do provide an opportunity to evaluate the effect of procedures on non-target impacts.

\section{Montpellier workshop on 'Evaluating indirect ecological effects of biological control'}

About 140 people from 26 countries participated in the Montpellier workshop (see IOBC, 1999, for a partial list of participants). The workshop was divided into two sessions. In the first session $(1.5 \mathrm{~h})$, participants were asked to develop and prioritize the major research questions concerning 
evaluation of non-target ecological effects of biological control. The participants were divided into 12 groups of $10-12$ people, with a facilitator and recorder for each group. The facilitators moderated the group discussions, ensuring that all had the opportunity to participate in the discussions. The recorders noted down the salient points raised. In the second session $(3 \mathrm{~h})$ participants were asked to develop methods for answering one of the high priority questions identified in the first session. Each group was assigned a single question chosen by the workshop facilitators (Robin Huettel, USDA, Washington, DC, and K.R. Hopper, USDA, Newark, Delaware) from among the 36 highest priority questions identified in first session. At the end of the workshop, the group facilitators described the results of each group's discussion.

\section{Session I: identification of major research questions}

The workshop identified questions on: (i) predicting host range; (ii) predicting non-target impacts; (iii) evaluating non-target impacts; (iv) evaluating risks versus benefits of pest management strategies; and (v) reducing non-target impacts. The points from these questions are summarized below, although not necessarily in question format.

\section{Predicting host range}

The general question here is how to predict host range of biological control agents from information gathered prior to introduction. Host range in the source region is routinely used to predict host range in the introduction region, but how reliable are such predictions? Systematic and ecological affinities between target and non-target species were identified as major factors that can affect whether the latter would be attacked. But the reliability of such affinities in predicting host range needs more research. Retrospective studies on past introductions would be particularly useful here. Quarantine studies with species from the introduction region provide a means of directly measuring attack on potential non-targets. However, differences between physiological/behavioural and ecological host ranges, as well as abnormal behaviour under laboratory conditions, present problems for interpretation of quarantine research. Furthermore, rearing and measurement of large numbers of potential non-target species in quarantine may be prohibitively expensive. How can the predictive power of quarantine studies be increased and the costs decreased? Perhaps a better understanding of underlying mechanisms (physiological, behavioural, ecological, genetic and phylogeographical) that determine host range would enhance the predictive power of host range measurements prior to introduction, whether done in the source region or in quarantine.

Little is known about the frequency, rapidity or mechanisms of evolutionary shifts in host range. Yet such knowledge is crucial in determining 
whether measurements of host range prior to introduction will reliably predict post-introduction host range. Furthermore, knowledge of evolutionary mechanisms of host range shifts might allow predictions of such shifts, at least in cases where intraspecific variation in the biological control agent was implicated. Lastly, the usefulness of systematic and ecological affinities among target and non-target species, mechanisms underlying host range and the likelihood of evolutionary shifts in host range are all likely to vary between herbivores, parasitoids, predators and pathogens of plants and insects, and among taxa within these functional groups. How much of the theory and techniques from one group can be applied to another group?

\section{Predicting non-target impacts}

Here the general question is how to predict non-target impacts prior to introduction of biological control agents. If such predictions were reliable, one could introduce only those natural enemies with little or no likely impact on non-target species. Some non-target species may be more vulnerable to impacts of natural enemies than others, so prediction of impacts might include characteristics of the non-target species as well as those of the candidates for introduction. The amounts of dispersal by biological control agents into habitats with non-target species will greatly affect the potential for non-target impact. Thus, methods are needed for predicting where and how much biological control agents will disperse after introduction. As with host range, behaviour in the source region may provide insights about dispersal and habitat distribution in the region of introduction. As with evaluating impacts after introduction, predicting impacts requires decisions about magnitude, spatio-temporal scale and the hierarchical level of impacts of concern. Propagation of indirect impacts through ecological systems is a major area where knowledge is lacking. Retrospective studies may provide information about the likelihood of such propagation for various sorts of biological control agents and target pests. Furthermore, retrospective studies with particular biological control agents may provide information about the safety of these agents for future introductions.

\section{Evaluating non-target impacts}

Here the general question is how to evaluate impacts on non-target species, given that an introduced biological control agent is already established. First, we must decide which non-target species to study. Should these be beneficial, rare, keystone, beautiful or some other subset of the species in the introduction region? Given that the list of potential non-target species may be large and a reduced set may be necessary, how can sentinel species be selected? Some non-target species may be particularly vulnerable to certain kinds of attack or to attack at certain life-stages, so the choice of non-target species to consider will vary with details of the potential 
interaction. Choosing non-target species of concern implicitly ignores impacts at higher levels of organization, e.g. communities or ecosystems. Given a set of non-target species, communities or ecosystems of concern, we must decide which impacts to measure. Impacts will vary with hierarchical level (individual, population, community, ecosystem) and spatio-temporal scale. Impacts may be direct through feeding, competition or other processes, or impacts may be indirect through effects on food, enemies or competitors of intervening species. Impacts may also include changes in life-history or genetics of non-target species. Furthermore, economic, social and political impacts may be involved. Lastly, some impacts may be unanticipated: how can we design monitoring systems for these? Measurement of impacts will require comparisons, e.g. between conditions pre- versus post-introduction or between sites with and without the biological control agent.

\section{Evaluating risks versus benefits}

What level of impact should be considered significant? Related to this question is that of the magnitude of benefits that biological control agents provide. How do we quantify the risks and benefits (economic, social, political, environmental) of biological control agents versus pesticides/ herbicides and other alternatives for pest management? What common currency can be used for weighing impacts versus benefits of various management options? If stricter protocols are used for biological control introductions, what will be the economic and social consequences?

\section{Reducing non-target impacts}

Given that introductions of biological control agents will always involve some risk of non-target impacts, how can such risk be minimized? Reliable predictions of impacts on target species would help reduce impacts on non-target species by providing control with fewer species introductions. But how can predictions of impacts on target species be improved? If a biological control agent has established and been found to have unacceptable non-target impacts, how can such impacts be mitigated?

\section{Session II: approaches to answering selected questions}

\section{How should we choose species to consider for non-target effects?}

Lists of species to study for non-target effects could be developed by exclusion. Phylogeny, morphology, physiology, behaviour, geography, climate, phenology and habitat could be used as exclusionary criteria to delineate a subset of species that would be most at risk from a given agent. Various systems (e.g. marine, freshwater, terrestrial, weeds, insect pests) and methods of control (augmentation, introduction) would require 
control agents. Long-term research on potential non-target species may improve the selection of species to study for non-target impacts.

\section{How do we predict and detect host range expansion?}

Lists of potential non-target hosts can be generated from literature data, field surveys and laboratory experiments. Ecological criteria for suitable hosts can be measured, including climate, potential natural enemies and competitors. Spatial analyses of data on distribution of the biological control agent in the source area could be used to predict where the agent is likely to occur in the target region. This information can be used to make statistical inferences about the potential for host range expansion. Predictions about host range should be tested with field studies of introduced agents. Here, one should collect data on population dynamics of the biological control agent and non-target species when together and separate (e.g. using cages, insecticides, geographical separation). Microcosms or plant assemblages could be used to study mechanisms of host range expansion. Various genetic strains of biological control agents and non-target species should be tested under a variety of ecological conditions (e.g. densities, alternative hosts) to determine the relative importance of ecological versus genetic constraints to host range. Lastly, if the molecular basis for host range were known, the likelihood of genetic changes in host range would be easier to infer.

\section{How can host range testing be improved?}

Considering only biological control by introductions and not augmentation or conservation, one could improve host range testing in the following ways. First, one should make results from host range testing available to others. Such results are often difficult to publish, but both positive and negative information about host range for each candidate is valuable, and should be made available either in the literature or in widely accessible databases so that it can be built upon. For each project, one should develop collaborative arrangements with others interested in the same targets and biological control agents or likely to be affected by the introductions. Such collaboration will minimize duplication, optimize resource use, overcome political boundaries, and thus both expedite and improve the safety of introductions. Because insects do not respect political boundaries, protocols should be harmonized among political jurisdictions that share biogeographical zones. At the beginning of each project, one should describe the systematics of the pest and biological control agents using the most advanced and appropriate methods available. Using information from the literature and from unpublished databases about similar taxa or ecological groups, one should rank the risks of non-target impacts of the biological control candidates. Using information on systematics and ecology, one should delineate actual host range in the source region and potential host range in the introduction region. 
Laboratory testing may also be required; this could involve choice or no-choice experiments that give yes/no or graded responses concerning which taxa are attacked. However, the limits of laboratory tests must be recognized, and they should take into account the ecology of the interaction, e.g. the effects of host plants on parasitism of herbivores and the factors likely to affect female oviposition behaviour. Experiments should account for the possibility of evolution in host range. One might use artificial selection to develop candidates likely to have maximum impact on the target and minimum impact on non-targets. Although laboratory tests measure interactions at the individual level, one should keep in mind how these affect dynamics at the population level. Data collected in these experiments should be linked to theory and used to develop explicit models to identify key parameters. If the results suggest a low likelihood of attack on non-target species, one should progress to semi-contained releases to study population-level processes. Assuming favourable results here, one could then proceed to introduction and post-establishment monitoring programmes. Such programmes should be mandated and should continue for sufficiently long to evaluate nontarget impacts. The results from these monitoring programmes should be made available in the literature or, if unpublished, in widely accessible databases.

How do we apply behavioural and physiological studies of host range in the real world?

A detailed answer to this question depends on the types of organisms; parasitoids and microbes would require different methodologies. However, a tiered approach should be used for any system. We already collect data on climate/microclimate, habitat/microhabitat and phenology of biological control agents and targets, as well as information on host finding, acceptability and suitability. We should collect such information for potential non-target species as well. This will reduce the risks of impacts on nontarget species, while also preventing rejection of safe agents that may, for example, be able to develop on a non-target species, but would never encounter it in the field.

\section{What constitutes acceptable risk in host range testing?}

Acceptability of risk is a societal issue, which may evolve with changing societal values and scientific knowledge. Determining the acceptability of risk requires cost-benefit analyses that should include ecological and ethical issues, as well as economics. The decisions themselves are not scientific. However, science can provide inputs to aid in the decision-making. For host range testing the crucial issue is how to translate measures of host range to levels of impact on non-target species. An agreed upon scale to rank severity of impacts would be useful. We also need to maximize the information content of host range testing. This includes designing studies 
with sufficient statistical power and ecological relevance. The species tested should provide new information because the goal is to reduce uncertainty, so that one should not, for example, test aphid parasitoids against bees when one knows very well that the bees will not be attacked. To relate results of host range testing to likely non-target impacts in the field, one should conduct ecological and economic studies of potential non-target species. These would reveal the vulnerability of non-target populations to attack by the candidate and, if so, whether there would be other ecological or economic consequences. Retrospective studies are needed to determine the relationship between past host range testing and actual non-target impacts in the field. Large differences in biota between source and target regions may reduce risk of non-target impacts. For agents introduced to control insects, where host range testing has not been as thorough as for introductions against weeds, retrospective studies of host ranges in source regions compared with those in target regions would be particularly useful. Some types of biological control agents (e.g. generalist predators) may always represent too great a risk of non-target impacts and thus might be black-listed. Lastly, host range testing should also be designed to reduce the risk of rejecting effective and safe agents.

\section{How can we predict spatio-temporal dispersion of biological control agents?}

Observations and experiments in source regions could be used to predict spatio-temporal distribution in introduction regions. Observations would include determination of phenology and geographical and habitat distributions, including climatic and ecological characteristics. Dispersal could be measured directly or inferred from measurements of flight capacity or molecular genetic analyses of population structure. Studies on how alternative hosts, natural enemies and competitors affect reproductive rates in various habitats would delineate factors influencing spatio-temporal distribution that might differ between source and introduction regions. Experimental release of populations in marginal environments of the source region would help to determine the limits to distribution and their causes. Experiments on temperature dependence of development could improve our understanding of phenology. Measurement of movement into artificial infestations of target pests and movement out when target pest populations crashed could be used to explore metapopulation dynamics. Mathematical models could be used to synthesize these data to make predictions about spatio-temporal distribution in target regions. However, such predictions would require detailed information about climate, habitats, alternative hosts, and potential natural enemies and competitors in target regions. Predictions made prior to introduction should be tested with observations and experiments after introduction. This would require collection of data similar to that described above for the source region. 
How can we determine the impacts on non-target species at the population level?

Assuming that non-target species of interest have already been identified, one should conduct long-term field studies of natural populations with and without the biological control agent. The studies should be long-term so that transient effects can be distinguished from more enduring effects. Comparison of non-target populations with and without the biological control agent could be achieved using pre- versus post-introduction dynamics, naturally occurring sites with and without the agent, or exclusion/removal manipulations. Each of these has problems, so it may be best to use a combination of them. The studies should be done in several ecoclimatic zones and replicated populations should be studied in each zone. To measure direct and indirect effects, one should follow the dynamics of the agent, a non-target species, and a natural enemy or competitor of the non-target species. Extension to more remote interactions would be too expensive. To reduce costs, one could begin by measuring incidence of attack. Above a chosen threshold of attack, one could start measuring population parameters. Sublethal as well as lethal effects may be important, but measurement of population-level impacts is the ultimate goal. Comparison of net reproductive rates with and without the agent over time would include sublethal effects and also address population-level impacts. If projections of further impacts were needed, data collected up to this point could be incorporated in mathematical models of population dynamics.

\section{What are the risks versus benefits of biological control when compared with other control methods?}

Three approaches could be taken to address this question: (i) a detailed case study or studies measuring all costs and benefits (ecological, social and economic) of all management strategies; (ii) prospective studies comparing in general the costs and benefits of various strategies and involving economists, sociologists and ecologists, as well as biological control researchers; and (iii) retrospective studies of past introductions to test how well costs and benefits were weighed. A major difficulty with detailed case studies is that measuring all costs and benefits of all management strategies may prove impossible or at least extremely difficult. Furthermore, a single case study or even several case studies would be difficult to generalize. Retrospective studies of introductions could indicate how risk-benefit decisions have been made. Such studies could address several questions: (i) What was known? (ii) What could have been known? (iii) Who made decisions and what was their expertise? (iv) What happened (i.e. what were the non-target impacts)? (v) What knowledge was gained (i.e. how were subsequent projects improved)? (vi) How do responses to the above questions vary with ecosystem, biogeographical province, political jurisdiction and decision-making framework? 
Introductions for control of weeds may be most amenable to such retrospective studies because detailed petitions are available which summarize pre-release studies and include host testing information. For any of these approaches, a variety of variables must be considered. These include human health risk, environmental risks, development costs, risk of pest resistance, sustainability, selectivity, compatibility with other management strategies, and distribution of costs and benefits across society.

How do we identify and achieve an ecologically valuable community after introduction of biological control agents?

This is a difficult question in part because defining an ecologically valuable community is difficult and to some extent a societal matter. However, drawing on community ecology, we can outline some steps that would address the question. First, the community of interest should be described before and after introduction of a biological control agent. There are various descriptive tools available, including indices of diversity, measures of stability and food web structure. Also, one should identify and prioritize at-risk taxa and bio-indicators of ecosystem processes. What constitutes a desired or valuable community then would need to be defined by consultation among various segments of society, through workshops, public opinion surveys, etc. These would allow delineation of goals, e.g. concerning biodiversity and ecosystem functions. Such delineation might require further taxonomic or ecological studies. The outcome would be a set of performance indicators, which might include, for example, desired population levels and genetic structure of sentinel species, food web structure and farm profitability. The state of these performance indicators could then be monitored after the introduction of biological control agents. Such monitoring could include studies of the population dynamics and genetics of sentinel species and perhaps studies of the dispersal and field behaviour of the biological control agents. If the performance indicators were not in the desired ranges, then various methods of mitigation could be used. Mitigation could be guided by mathematical models developed from the information gathered during pre- and post-introduction monitoring.

How can we use retrospective studies to predict the safety of future releases?

The practice of biological control in general and of predicting non-target impacts in particular could be greatly improved by formulating testable hypotheses and then testing them. Examples of such hypotheses are:

1. The more intense the host range screening, the more predictable the non-target impacts;

2. Host specificity testing works for predicting direct effects but not for predicting indirect effects; 
3. Direct effects are more likely for non-target species that are closely related to the target;

4. The more closely related non-target species are to target species, the more likely are non-target impacts;

5. Less specific biological control agents have more non-target impacts than more specific agents;

6. Non-target species in simpler communities are more at risk than those in complex communities;

7. Non-target impacts are a function of the inherent host ranges of biological control agents rather than other factors;

8. Effective biological control agents have fewer non-target impacts than ineffective agents.

To test these hypotheses, one should study a random subset of past projects, stratified using an ecological framework, e.g. by time since introduction, release rate and pattern, number of species introduced, number of species closely related to the target, community complexity and amount of host range testing done. The literature (e.g. Julien and Griffiths, 1998) and unpublished databases (e.g. BIOCAT, ROBO) would at least provide information about these variables and perhaps some information on non-target impacts. However, most of the data on non-target impacts would have to be collected in field surveys and experiments. Data on abundance, attack, mortality, and the biotic and abiotic environment could be collected in field surveys at the release points, the edge of current biological control agent distribution and beyond its current distribution. Collection of similar data in the source region might also prove useful. These data would need to be supplemented with results from field experiments to evaluate the actual impact of biological control agents on non-target species.

\section{Conclusions}

Although many of the same people contributed to both workshops, they represented a wide variety of viewpoints concerning potential for non-target impacts from biological control introductions. Both workshops highlighted the need for retrospective studies to: (i) identify cases of significant non-target impacts; (ii) explore mechanisms involved when such impacts are found; (iii) evaluate the reliability of host range testing protocols; and (iv) determine whether and under what circumstances host ranges of biological control agents have evolved subsequent to introduction. The workshops emphasized the need for better understanding of the mechanisms underlying host specificity to improve accuracy of predictions of host range. Although participants recognized the need to identify which non-target species and hierarchical levels of damage should be studied, they also agreed that such identification involves societal as well as scientific issues. Finally, the workshops recognized the desirability 
of collaboration among ecologists, economists, sociologists and biological control practitioners in research to assess and reduce the potential for non-target impacts of biological control introductions.

\section{References}

Andres, L.A. (1985) Interactions of Chrysolina quadrigemina and Hypericum spp. in California. In: Delfosse, E.S. (ed.) Proceedings of the Sixth International Symposium on Biological Control of Weeds. Agriculture Canada, Ottawa, pp. 235-239.

Blossey, B., Schroeder, D., Hight, S.D. and Malecki, R.A. (1994) Host specificity and environmental impact of two leaf beetles (Galerucella calmariensis and G. pusilla) for biological control of purple loosestrife (Lythrum salicaria). Weed Science 42, 134-140.

Carruthers, R.I. and Onsager, J.A. (1993) Perspective on the use of exotic natural enemies for biological control of pest grasshoppers (Orthoptera: Acrididae). Environmental Entomology 22, 885-903.

Diehl, J. and McEvoy, P.B. (1990) Impact of cinnabar moth (Tyria jacobaeae) on Senecio trianularis, a non-target native plant in Oregon. In: Delfosse, E.S. (ed.) Proceedings of the Seventh International Symposium on Biological Control of Weeds. Ministero dell'Agricoltura e delle Foreste and CSIRO, Rome, pp. 119-126.

Duan, J.J., Ahmad, M., Joshi, K. and Messing, R.H. (1997) Evaluation of the impact of the fruit fly parasitoid Diachasmimorpha longicaudata (Hymenoptera: Braconidae) on a nontarget tephritid, Eutreta xanthochaeta (Diptera: Tephritidae). Biological Control 8, 58-64.

Hasan, S. and Delfosse, E.S. (1995) Susceptibility of the Australian native, Heliotropium crispatum, to the rust fungus Uromyces heliotropii introduced to control common heliotrope, Heliotropium europaeum. Biocontrol Science and Technology 5, 165-174.

Hill, M.P. and Hulley, P.E. (1995) Biology and host range of Gratiana spadicea (Klug, 1829) (Coleoptera, Chrysomelidae, Cassidinae), a potential biological control agent for the weed Solanum sisymbriifolium Lamarck (Solanaceae) in South Africa. Biological Control 5, 345-352.

IOBC (1999) Evaluating indirect ecological effects of biological control. IOBC-WPRS Bulletin 22.

Julien, M.H. and Griffiths, M.W. (eds) (1998) Biological Control of Weeds: a World Catalog of Agents and their Target Weeds. CAB International, Wallingford, UK.

Kok, L.T., McAvoy, T.J., Malecki, R.A., Hight, S.D., Drea, J.J. and Coulson, J.R. (1992) Host specificity tests of Galerucella calmariensis (L.) and G. pusilla (Duft.) (Coleoptera: Chrysomelidae), potential biological control agents of purple loosestrife, Lythrum salicaria L. (Lythraceae). Biological Control 2, 282-290.

LaSalle, J. and Gauld, I.D. (1992) Parasitic Hymenoptera and the biodiversity crisis. Redia 74, 315-334.

Louda, S.M., Kendall, D., Connor, J. and Simberloff, D. (1997) Ecological effects of an insect introduced for the biological control of weeds. Science 277, 1088-1090.

Malecki, R.A., Blossey, B., Hight, S.D., Schroeder, D., Kok, L.T. and Coulson, J.R. (1993) Biological control of purple loosestrife. Bioscience 43, 680-686. 
Marohasy, J. (1996) Host shifts in biological weed control: real problems, semantic difficulties or poor science? International Journal of Pest Management 42, 71-75.

McEvoy, P.B. (1996) Host specificity and biological pest control. Bioscience 46, 401-405.

Olckers, T., Zimmermann, H.G. and Hoffmann, J.H. (1995) Interpreting ambiguous results of host-specificity tests in biological control of weeds - assessment of 2 Leptinotarsa species (Chrysomelidae) for the control of Solanum elaeagnifolium (Solanaceae) in South Africa. Biological Control 5, 336-344.

Ricciardi, A., Neves, R.J. and Rasmussen, J.B. (1998) Impending extinctions of North American freshwater mussels (Unionoida) following the zebra mussel (Dreissena polymorpha) invasion. Journal of Animal Ecology 67, 613-619.

Simberloff, D. and Stiling, P. (1996) How risky is biological control? Ecology 77, 1965-1974.

Turner, C.E., Pemberton, R.W. and Rosenthal, S.S. (1987) Host utilization of native Cirsium thistles (Asteraceae) by the introduced weevil Rhinocyllus conicus (Coleoptera: Curculionidae) in California. Environmental Entomology $16,111-115$.

Unruh, T.R. and Messing, R.H. (1993) Intraspecific biodiversity in Hymenoptera: implications for conservation and biological control. In: LaSalle, J. and Gauld, I.D. (eds) Hymenoptera and Biodiversity. CAB International, Wallingford, UK, pp. 27-52.

Willis, A.J., Ash, J.E. and Groves, R.H. (1995) The effects of herbivory by a mite, Aculus hyperici nutrient deficiency on growth in Hypericum species. Australian Journal of Botany 43, 305-316. 